\title{
He I D3 OBSERVATIONS OF THE 1984 MAY 22 M6.3 SOLAR FLARE
}

\author{
Chang Liu $^{1}$, Yan Xu $^{1}$, Na Deng $^{1}$, Jeongwoo LeE $^{1,2}$, Jifeng Zhang $^{1,3}$, Debi Prasad Choudhary ${ }^{4}$, And Haimin Wang ${ }^{1}$ \\ ${ }^{1}$ Space Weather Research Laboratory, Center for Solar-Terrestrial Research, New Jersey Institute of Technology, \\ University Heights, Newark, NJ 07102-1982, USA; chang.liu@njit.edu \\ ${ }^{2}$ School of Space Research, Kyung Hee University, Yongin 446-701, Korea \\ ${ }^{3}$ Department of Mechanical and Industrial Engineering, New Jersey Institute of Technology, University Heights, Newark, NJ 07102-1982, USA \\ ${ }_{4}^{4}$ Physics and Astronomy Department, California State University Northridge, 18111 Nordhoff Street, Northridge, CA 91330-0001, USA \\ Received 2013 April 12; accepted 2013 June 25; published 2013 August 16
}

\begin{abstract}
The He I D3 line has a unique response to a flare impact on the low solar atmosphere and can be a powerful diagnostic tool for energy transport processes. Using images obtained from the recently digitized films of the Big Bear Solar Observatory, we report D3 observations of the M6.3 flare on 1984 May 22, which occurred in an active region with a circular magnetic polarity inversion line (PIL). The impulsive phase of the flare starts with a main elongated source that darkens in D3, inside of which bright emission kernels appear at the time of the initial small peak in hard X-rays (HXRs). These flare cores subsequently evolve into a sharp emission strand lying within the dark halo; this evolution occurs at the same time as the main peak in HXRs, reversing the overall source contrast from $-5 \%$ to $5 \%$. The radiated energy in D3 during the main peak is estimated to be about $10^{30} \mathrm{erg}$, which is comparable to that carried by nonthermal electrons above $20 \mathrm{keV}$. Afterward, the flare proceeds along the circular PIL in the counterclockwise direction to form a dark circular ribbon in D3, which apparently mirrors the bright ribbons in $\mathrm{H} \alpha$ and $\mathrm{He}$ I $10830 \AA$. All of these ribbons last for over one hour in the late gradual phase. We suggest that the present event resembles the so-called black-light flare that was proposed based on continuum images, and that D3 darkening and brightening features herein may be due to thermal conduction heating and the direct precipitation of high-energy electrons, respectively.
\end{abstract}

Key words: Sun: activity - Sun: chromosphere - Sun: flares - Sun: magnetic fields - Sun: X-rays, gamma rays

Online-only material: color figures, animations

\section{INTRODUCTION}

$\mathrm{H} \alpha$, the most commonly used line in solar observations, is broad and optically thick and responds to a wide range of features in the solar chromosphere. In contrast, the He I D3 line, located at $5876 \AA$ near the Na I doublet D1 and D2, is narrow and mostly optically thin and is sensitive to nonthermal excitation. For the latter reason, the D3 line is considered better suited to the study of higher energy phenomena than $\mathrm{H} \alpha$. The most unique property of D3 is that it appears in absorption in surges, eruptive filaments, flare ejecta, and weak flares, and turns into emission only in intense flares. Compact and transient bright cores or strands are often observed in D3 during the impulsive phase of major flares, displaying a close spatiotemporal association with footpoint-like white light (WL) and hard X-ray (HXR) emission (e.g., Labonte 1979; Zirin 1980; Zirin \& Neidig 1981; Feldman et al. 1983; Tanaka \& Zirin 1985; Zirin \& Tang 1990; Wang et al. 1996). D3 emission can reach about twice the photospheric intensity during flares; this result means that the medium has a high temperature $\left(\sim 2 \times 10^{4} \mathrm{~K}\right)$ and high density $\left(\sim 10^{13} \mathrm{~cm}^{-3}\right)$ flaring plasma. Therefore, D3 may provide a diagnostic of the main flare energy source in the low atmosphere, complementary to WL and HXRs, as well as serving as a sensitive probe for tracing the flare development. The simultaneity of D3 and HXR footpoint emission could indicate that accelerated electrons penetrate down to at least the low level, as indicated by the above density (Zirin 1988). Wang et al. (1996) observed during the initial HXR peak of an M2.7 flare that D3 emission only occurred at one of the HXR footpoints with a harder spectrum. Another X12 flare studied by Tanaka \& Zirin (1985) started with multiple compact brightenings in D3 and WL, two of which grew to the main flare footpoints when the HXR spectrum hardened with a sharply increasing flux. The footpoints further developed into two ribbons in D3 above sunspots around the soft $\mathrm{X}$-ray (SXR) peak, mainly due to heating from the hot thermal plasma that is conducted down to dense layers. The D3 line has been dubbed "a gold mine of information" on flares (Zirin 1988); nonetheless, it has been largely ignored in recent flare observations.

As one of the strongest $\mathrm{He}$ lines in the visible spectrum, the D3 line results from transitions between the $2 p^{3} P$ and $3 d^{3} D$ terms of the He I triplet (ortho-helium), which involves a metastable level about $20 \mathrm{eV}$ above the ground state. One mechanism to populate the metastable level is that the coronal extreme ultraviolet (EUV) radiation ionizes neutral $\mathrm{He}$ atoms primarily in the singlet ground state at typical chromospheric conditions, and subsequent recombinations produce a larger population of $\mathrm{He}$ in the triplet systems, hence strengthening the D3 feature (e.g., Mauas et al. 2005; Centeno et al. 2008). This photoionization-recombination (PR) scenario is supported by observations of the bright D3 band in a narrow height range of 1000-2000 km above the solar limb; these observations are, however, not reported over coronal hole regions (Zirin 1975). We note that EUV photons only help in populating the triplet levels, which then subsequently radiate by scattering the photospheric emission. Thus, in order for D3 to turn into emission against the disk, direct collisional excitation (CE) must exceed the photospheric radiative excitation. It can be deduced from this requirement that at $T \approx 2 \times 10^{4} \mathrm{~K}$, the transition from absorption to emission of D3 in a flare would occur as the density of the 
heated atmosphere reaches $N \gtrsim 5 \times 10^{12} \mathrm{~cm}^{-3}$ (Zirin 1988). Thus far, only a single event exhibiting this implied reversal of D3 intensity has been reported. It was the SOL1978-0710T17:35 $\mathrm{M} 8$ flare, in which a dark ring (circular ribbon) in D3 around a satellite spot in the preflare state immediately preceded two bright D3 emission kernels, which supposedly lay below the ring (Zirin 1980).

Intriguingly, such an evolution from absorption to emission is very reminiscent of the so-called "negative flares" on stars, in which a preflare dip in the continuum level, i.e., a $~ 20 \%$ decrement in amplitude in a time period of seconds to minutes, is seen right before the continuum brightening (e.g., Hawley et al. 1995, and references therein). Henoux et al. (1990) termed such a phenomenon "black-light flares" (BLFs) and proposed that continuum BLFs could also happen on the Sun for some $20 \mathrm{~s}$ before WL flares (WLFs). The underlying mechanism could be that the nonthermal ionization of hydrogen by the bombardment of electron beams on a cool atmosphere first produces an increase of $\mathrm{H}^{-}$opacity, and its diminutive effect is overtaken by the enhanced emissivity from a fully heated atmosphere near the flare maximum phase (Grinin 1983; Henoux et al. 1990; Ding \& Fang 2000; Ding et al. 2003). Observations and studies of BLFs would certainly be important for solar and stellar flare physics, as further information could be obtained on the heating of the low atmosphere in the flaring process. Nevertheless, solar BLFs in the continuum are extremely rare. van Driel-Gesztelyi et al. (1994) surveyed all nine Yohkoh WLFs greater than GOESclass M6 but found no unambiguous evidence for the BLF association. A possible example presented by Henoux et al. (1990) is a negative intensity contrast up to $\sim 5 \%$ in the $5500 \AA$ continuum a few minutes before the SOL1981-07-26T13:53 X3 WLF. Another candidate is the SOL2001-03-10T04:05 M6.7 WLF observed near the Ca II $8542 \AA$ line, for which Ding et al. (2003) showed a dip of $\sim 1 \%-2 \%$ in its early phase before the continuum emission.

Nonthermal electron beams cannot only lead to BLFs in the continuum, but also are able to drastically change spectral lines. Using non-LTE calculations, Ding et al. (2005) demonstrated that in the nonthermal case, collisional ionization followed by recombinations $(\mathrm{CR})$ may significantly contribute to populating the He triplet levels corresponding to the He I $10830 \AA$ line; this contribution acts in addition to the PR and CE mechanisms. Importantly, the influence of CR on the $10830 \AA$ line, when compared to the case without the nonthermal effects, was modeled by the authors. In their result, the nonthermal effect can cause stronger absorption early in a flare and stronger emission at the flare maximum. We consider that such a transition from absorption to emission of spectral lines, if observed, could be regarded as a variant of BLFs analogous to those in the continuum. In this context, the aforementioned event of a preflare dark ring followed by bright kernels (Zirin 1980) turns out to be a very rare instance of D3 BLFs. To our knowledge, no study has been carried out about the nonthermal effects on the He I D3 line.

In this paper, we present a detailed analysis of the SOL198405-22T15:03 M6.3/2B flare, taking advantage of the unique D3 observation made at the Big Bear Solar Observatory (BBSO). The most prominent characteristics of this event include (1) the main D3 flare source first darkens in intensity and then changes to emission at the time of the HXR peak, making it an excellent candidate for a BLF and (2) in the late phase, two dark ribbons

\footnotetext{
5 Following the IAU solar target naming convention (Leibacher et al. 2010).
}

form in D3, mirroring their bright counterparts in $\mathrm{H} \alpha$ as well as He I $10830 \AA$. We also compare the radiated energy in D3 with the energy carried by electron beams at the flare peak time and discuss the event progression using $\mathrm{H} \alpha$ images. The outline of the paper is as follows: in Section 2, we describe the data sets and the reduction procedure. In Section 3, we present the main results of data analysis and discuss their implications. Major findings are summarized in Section 4.

\section{OBSERVATIONS AND DATA REDUCTION}

The BBSO has a long tradition of flare observations at $\mathrm{H} \alpha$ wavelengths. In addition, the BBSO started in 1973 to take high-resolution images in the D3 line using 25 or $65 \mathrm{~cm}$ telescopes (Feldman et al. 1983). All of the BBSO data obtained before 1995 were recorded on $35 \mathrm{~mm}$ films, a large portion of which have recently been digitized (see Wang \& Liu 2012 for a more complete description). The film data have also been publicly released, ${ }^{6}$ which opens the door for studying a wealth of historical solar phenomena. The present event (SOL198405-22T15:03 M6.3) is selected from our survey on D3 flare observations.

We mainly used the digitized partial-disk D3 and $\mathrm{H} \alpha$ images taken by the east and west benches, respectively, of the $25 \mathrm{~cm}$ telescope, with a pixel size of $\sim 0^{\prime} .15$ and a cadence of $\sim 15 \mathrm{~s}$. These images are capable of offering subarcsecond resolution when the seeing conditions at the BBSO are excellent (as in the later phase of the flare event under study). A non-linear conversion table supplied by the digitizer, which transforms the original 12 bit digitized data to 8 bit, was applied to deal with the nonlinear intensity response of films. The image alignment was implemented with sub-pixel precision and the intensity was normalized to that outside the flaring region in a quiet-Sun area. A de-stretching algorithm (Shine et al. 1994) using running references was applied in order to reduce the atmospheric distortion. We define the image contrast as $\left(I-I_{0}\right) / I_{0}$, where $I$ is the intensity of the feature of interest and $I_{0}$ is the intensity of the same feature in the preflare state or that of the undisturbed quietSun background. For the $\mathrm{H} \alpha$ image time series, we computed the intensity-weighted centroid positions of the flare ribbons and used the relative distance of source centroids to infer the progression of the flare (e.g., Liu et al. 2010). Additional auxiliary data used in our study include the digitized full-disk $\left(\sim 1^{\prime \prime} .4\right.$ pixel $\left.^{-1}\right)$, one minute cadence $\mathrm{H} \alpha$ images taken by the National Solar Observatory (NSO) at Sacramento Peak (SP) and the daily full-disk spectroheliogram observation at $10830 \AA$ made by the NSO at Kitt Peak (KP) using its 512-channel magnetograph (Livingston et al. 1976).

The daily full-disk magnetic field structure of the photosphere in the line of sight (LOS) was also observed by the NSO/KP in Fe I $8688 \AA$ A. We registered the D3 and $\mathrm{H} \alpha$ images with the NSO/ KP magnetogram by matching sunspot and plage areas, with an alignment accuracy estimated to $\lesssim 5^{\prime \prime}$. To reveal the magnetic structure of the flaring region, we resorted to the potential field extrapolation in Cartesian coordinates.

$\mathrm{X}$-ray observations were used to study the temporal evolution of the flare and understand the nature of the D3 emission. SXR and HXR time profiles were obtained with the GOES 5 satellite and the Hard X-Ray Burst Spectrometer (HXRBS; Orwig et al. 1980), respectively, the latter of which was on board the Solar Maximum Mission (SMM). We also utilized HXR spectra fitted to all 15 channels of the HXRBS covering the

\footnotetext{
6 http://sfd.njit.edu
} 


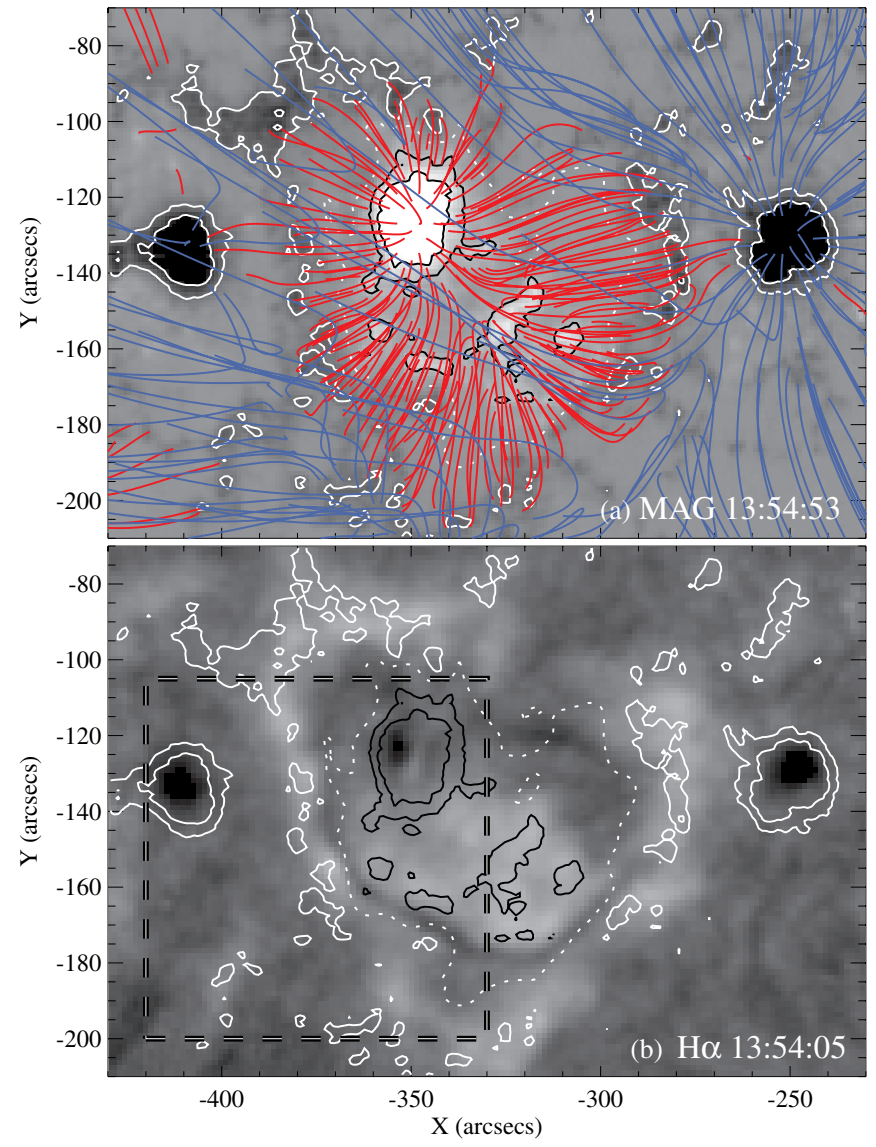

Figure 1. Magnetic structure of the flaring region in the leading portion of NOAA AR 4492. (a) A NSO/KP magnetogram (scaled from -800 to $800 \mathrm{G}$ ) overplotted with the PIL (dotted line), and the closed (red) and open (blue) field lines extrapolated using a potential field approximation. The levels of the magnetic field contours are \pm 300 and $\pm 700 \mathrm{G}$. (b) A preflare NSO/SP H $\alpha$ image overplotted with the same magnetic contours as well as the PIL. The dashed box indicates the FOV of Figure 3. All the images in this paper are aligned with respect to 1984 May 22 14:50 UT.

(A color version of this figure is available in the online journal.)

energy range of 24-400 keV; these spectra were computed by an automatic spectral fitting routine using a single power-law model (Kiplinger 1995). The derived spectral parameters allowed us to evaluate the nonthermal thick-target electron power above a certain low-energy cutoff (Brown 1971). In addition, the SXR images were available from the X-Ray Polychromator (XRP; Acton et al. 1980) on board the SMM.

\section{RESULTS AND ANALYSIS}

In this section, we first provide a brief account of the magnetic field structure of the flaring region in the preflare state. We then concentrate on describing the characteristic flare activities in D3, $\mathrm{HXR}$, as well as $\mathrm{H} \alpha$ wavelengths throughout the event. More dynamic detail can be seen in the accompanying animations in the online journal. ${ }^{7}$

\subsection{Magnetic Field Structure}

The active region (AR) of interest NOAA AR 4492 started to appear in the $\beta \gamma \delta$ magnetic configuration from 1984 May 19 and showed growth until May 21. The AR decayed in size from

\footnotetext{
7 For maximal use of the field of view (FOV), the images in the animations were not corrected for the solar $p$-angle.
}

May 22, when the present M6.3/2B flare occurred in the leading portion of the AR with a $\beta$ configuration (see Figure 1(a)). A prominent property of magnetic field structure of this flaring region is that the central spot of positive polarity seems to be encircled by a surrounding negative field, forming a quasicircular magnetic polarity inversion line (PIL; the dotted line in Figure 1). This circular PIL is well delineated by the circle-like $\mathrm{H} \alpha$ filament, which lies closely along the PIL (Figure 1(b)). Different from classical eruptions, this filament does not show a preflare activation and largely survived after the flare, as is clearly seen in the time-lapse $\mathrm{H} \alpha$ movies. This feature may indicate that the flare-associated magnetic reconnection could occur above the filament (e.g., Liu et al. 2007).

Remarkably, the result of the potential field extrapolation model (Figure 1(a)) reveals dome-shaped closed fields (red) overarching the filament. The closed dome is enveloped by open field lines (blue) stemming from the surrounding regions. The overall structure is highly indicative of a fan-spine configuration, which is usually associated with a coronal null point. It has been found that three-dimensional (3D) magnetic reconnection could occur at such a null point (Lau \& Finn 1990; Török et al. 2009), especially when pronounced asymmetry is present (as for this AR; see Figure 1(a); Pariat et al. 2010). It is also found that sometimes flares associated with such 3D reconnection may exhibit a central compact kernel situated within an extended circular flare ribbon (e.g., Masson et al. 2009). In fact, observational signatures of the present flare bear much resemblance to those described in this scenario, and we will discuss these signatures in more detail in Section 3.3 below.

\subsection{Event Evolution in D3}

\subsubsection{A BLF}

According to the GOES and HXRBS X-ray fluxes, the event started at 14:51 UT and peaked at 15:03 UT on 1984 May 22, and had four conspicuous HXR spikes p1-p4 (see Figure 2(a)). The initiation of the flare in D3 images is marked by a gradual darkening of the elongated main source S1 above the weak positive magnetic field region (cf. Figures 3(a)-(c) and (f)). It is notable that within this darkening source S1, two D3 kernels begin to brighten from $\sim 14: 54$ :30 UT (Figures 3(c) and (d)), cotemporal with the first HXR spike p1. The two bright kernels then appear to extend in a direction parallel to the PIL, forming an emission strand lying within the dark halo-like structure at 14:57:05 UT (Figure 3(e)), which is simultaneous with the most intense spike p2 in HXRs from $24-400 \mathrm{keV}$. An intriguing observation is that the strand apparently reaches its peak brightness a little later at 14:57:20 UT (Figure 3(f)), at the time of a slightly harder HXR spectrum (Figure 2(c)). From $\sim$ 14:57:50 UT, S1 broadens and enhances, particularly in its northern end, and during the time period of the HXR spike p3, S1 spreads eastward into the sunspot region (Figures 3(g)-(i)).

To more quantitatively assess the temporal relationship between D3 emission and flare energy release, we measure the mean contrast of the D3 source $\mathrm{S} 1$ in a boxed region (see Figure 3(f)) and draw its time profile in Figure 2(b) (black line). It can be unambiguously seen that $\mathrm{S} 1$ first darkens and dips in intensity, reaching a contrast amplitude of $-5 \%$ in about 4 minutes from 14:52 to $14: 56$ UT despite the brightening kernels. It then quickly turns to positive contrast after being overtaken by the bright emission strand and peaks with an enhancement of $\sim 5 \%-10 \%$ concurrently with the HXR spikes p2 and p3 with hardened spectra. It is worth mentioning that the highest D3 


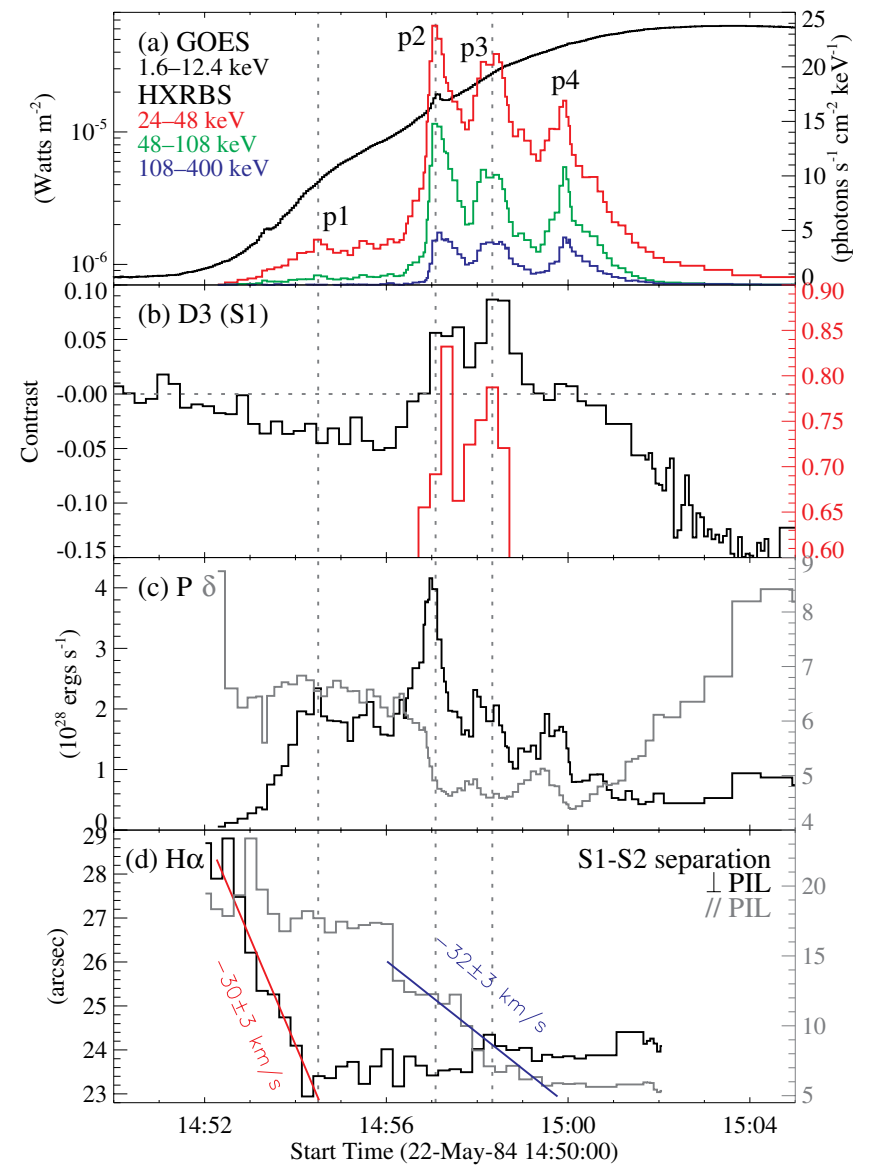

Figure 2. Multiwavelength temporal evolution of the flare. (a) GOES SXR flux overplotted with HXRBS photon fluxes. The 48-108 and 108-400 keV fluxes are multiplied by 10 and 30, respectively. (b) Mean intensity contrast (black) of the D3 source S1 (the boxed region in Figure 3(f)) and that of its brightest core region with a contrast enhancement greater than 0.6 (red). (c) Thick-target electron spectral index (gray) and electron power for a low energy cutoff of $20 \mathrm{keV}$ (black). (e) Separation of $\mathrm{H} \alpha$ ribbons $\mathrm{S} 1$ and $\mathrm{S} 2$ in the directions perpendicular and parallel to the PIL.

(A color version of this figure is available in the online journal.)

intensity of S1 actually occurs at the time of the strongest HXR peak p2, as expected. This peak D3 intensity shows a contrast up to $100 \%$, as previously observed in other flares of similar magnitude (e.g., Zirin 1980). This result is clear in the time profile of averaged contrast that shows only contrasts greater than $60 \%$ (red line in Figure 2(b)). Here, the most significant result is the intensity reversal of $\mathrm{S} 1$ from absorption to emission, which qualifies this event as a rarely observed BLF. For the first time, this observation provides direct evidence for the unique property of BLFs in the D3 line.

We note that the absorption feature in D3 was generally interpreted as being due to heating of the dense layer by thermal conduction (Zirin 1988). This hypothesis could explain the darkening of S1 below the BLF level in the later thermal phase (after 15:02 UT). More importantly, D3 and HXR emission in the earlier impulsive phase are simultaneous with a good correlation, which implies electron beam heating, as suggested before (Feldman et al. 1983; Zirin 1988). In other words, D3 emitting sources in the flare impulsive phase could indicate the location of electron precipitation. As further evidence of this hypothesis, a compact and weak D3 bright kernel S2 is located at the umbral region of the western sunspot with negative polarity. S2 becomes visible after $\sim 14: 56 \mathrm{UT}$, peaks at the HXR spike p1 around 14:57 UT with a contrast of $\sim 70 \%$ against the dark sunspot, and propagates southward after $\sim 14: 58 \mathrm{UT}$ (Figures 3(d)-(i)). We speculate that S1 and S2 are a pair of conjugate sources at the feet of reconnecting flare loops, which are indicated by the loop-like structure in SXRs crossing over the PIL (contours in Figure 3(m)). As S2 lies within the stronger umbral field region, its compactness in D3 could be due to the magnetic mirroring effect (e.g., Kundu et al. 1995). We also note that the last HXR spike p4 at 15:00 UT is reflected in D3 as a newly formed source S3 in the south (Figure 3(j)). Flaring loops could connect $\mathrm{S} 2$ and $\mathrm{S} 3$ at this stage, as imaged in SXRs at a later time (Figure 3(o)).

\subsubsection{Bright Core-Dark Halo Structure}

We placed two slits perpendicular to the elongated source $\mathrm{S} 1$, as shown in Figure 3; the intensity profiles along these slits are plotted in Figure 4 in order to more closely examine the dark absorption and bright emission features in D3 and their temporal evolution. Gaussian fits were applied to estimate the characteristic sizes and peak positions of the intensity profiles.

Slit 1 crosses the maximum of the D3 kernel in Figure 3(d). The intensity profile along it already displays a discernible darkening about eight minutes before the flare (gray line), possibly implying the occurrence of preflare heating. After the flare onset at 14:52:20 UT, the darkening contrast deepens and shifts toward the PIL (green line). The later bright emission kernel at 14:56:35 (blue line) and 14:57:20 UT (red line) peaks $\sim 4^{\prime \prime}$ to the east. However, the darkening contrasts on either side of the emission peak both deepen consecutively with a step of $\sim 0.05 \%$. Furthermore, the western darkening keeps moving toward the PIL by $\sim 4^{\prime \prime}$. Additionally, while the eastern darkening broadens, the western darkening narrows at the HXR peak time (red line) to reach the largest contrast of about $-3 \%$. It is reasonable to surmise that the preference of D3 darkenings toward the PIL is caused by the enhanced density near the filament lying along the PIL region (see Figure $3(\mathrm{k})$ ), which provides a favorable condition for D3 line formation. These results thus clearly illustrate a D3 flare source structure comprising a low-lying bright emission core (due to nonthermal beam heating) encompassed by darkening halos (due to thermal conduction heating). Consistent with this picture, the $\mathrm{H} \alpha$ intensity profile along the slit appears, in contrast, as a broad bump (the central part is saturated) with about twice the FWHM width, straddling the D3 bright core and dark halos. It is also appealing to suggest that the bright core-dark halo source structure in D3 is analogous to the corehalo morphology of WLF sources in the photospheric level (Xu et al. 2006), where both the core and halo are bright and might be produced by direct electron heating and chromospheric backwarming, respectively.

Slit 2 crosses the maximum of the D3 strand S1 around the HXR peak time (Figure 3(f)). Its central region first darkens (green line), then reverts back (blue and red lines) to become a very intense emission core peaking at $100 \%$ contrast while with a similar FWHM compared to the case of slit 1. It does not seem that a dark halo developed in the east of the core; nevertheless, a darkening region is similarly built up in the western region close to the PIL/filament.

\subsubsection{Flare Energy Release}

We further chose flare peak p2 in D3 and HXRs, which is well defined both temporally and spatially, to roughly evaluate the energy budget. The underlying assumption is that the D3 


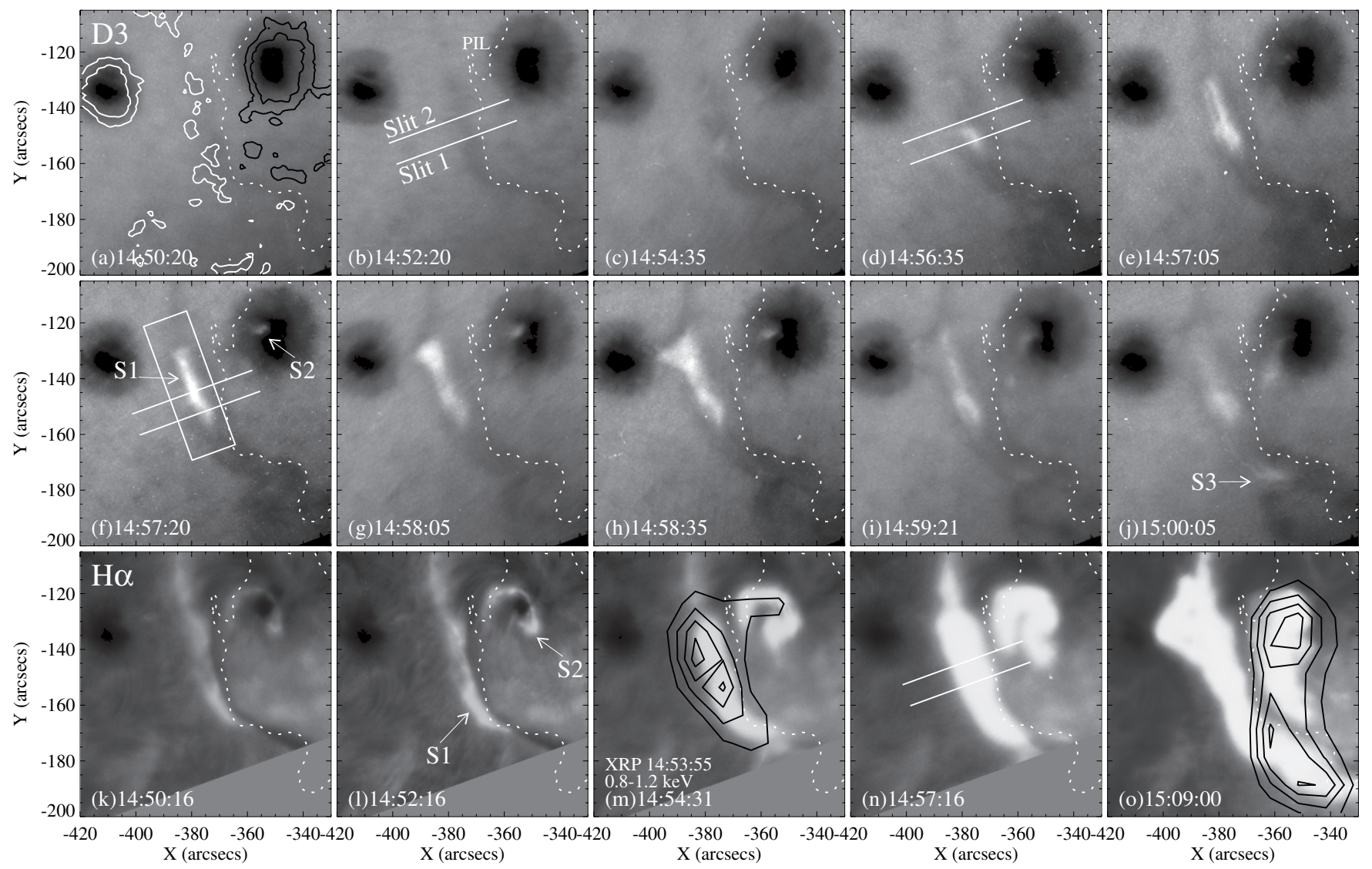

Figure 3. Time sequence of $\mathrm{D} 3(\mathrm{a}-\mathrm{j})$ and $\mathrm{H} \alpha(\mathrm{k}-\mathrm{o})$ images during the flare impulsive phase. The box in (f) marks the area used for calculating the overall light curve of the D3 source S1 (black) in Figure 2(b). The white lines in (b), (d), (f), and (n) are slits perpendicular to the flare ribbons/strands, along which the intensity profiles are drawn in Figure 4. Slits 1 and 2 pass through the D3 source maxima in (d) and (f), respectively. The black contours (30\%, 50\%, 70\%, and 90\% of the maximum flux) in (m) and (o) represent the XRP SXR images. The magnetic field contours in (a) are the same as those in Figure 1 and the dotted line is the PIL. The image FOV is denoted by the dashed box in Figure 1(b).

(Animations of this figure are available in the online journal.)

line emission could be optically thick in flares (Svestka 1976; Zirin 1988), hence the gas radiates with a source function close to the Planck function for the corresponding temperature. In the canonical flare model (e.g., Wang et al. 1998), the intensity contrast enhancement of a flare is given by:

$$
\frac{\Delta I}{I}=\frac{B(T+\Delta T, \lambda)-B(T, \lambda)}{B(T, \lambda)},
$$

where $B$ is the Planck function and $\Delta T$ is the temperature perturbation due to flare heating. The flare radiative loss flux can then be written as:

$$
\Delta L=4 \sigma T^{3} \Delta T \operatorname{erg} \mathrm{cm}^{-2} \mathrm{~s}^{-1},
$$

where $\sigma$ is the Stefan-Boltzmann constant (Najita \& Orrall 1970 ). For the $n \approx 10^{13} \mathrm{~cm}^{-3}$ that is required for $\mathrm{D} 3$ emission, $T$ is about $6400 \mathrm{~K}$ according to the VAL-F atmospheric model (Vernazza et al. 1981). We use this model because it was constructed for the magnetic network regions suitable for the present flare, hence is better than other models for the quiet-Sun conditions. If we integrate $\Delta L$ over the source $\mathrm{S} 1$ and adopt a duration of $30 \mathrm{~s}$, the radiative energy in D3 during peak $\mathrm{p} 2$ is estimated to be about $1.3 \times 10^{30} \mathrm{erg}$. This energy is comparable to the nonthermal energy $E_{\text {nonth }} \approx 1.2 \times 10^{30}$ erg released by the thick-target electrons greater than $20 \mathrm{keV}$ around peak p2 (Figure 2(c)). Obviously, such a comparison is meaningful provided that electrons with initial energies of $20 \mathrm{keV}$ and above can penetrate to densities of $\sim 10^{13} \mathrm{~cm}^{-3}$. A simplified equation relating the observed photon energy $\epsilon$ to the column density $N$ of a certain layer, into which the parent electrons of energy $E$ can precipitate, is:

$$
N=10^{20} \mathrm{~cm}^{-2}\left(\frac{\epsilon}{20 \mathrm{keV}}\right)^{2}
$$

(Brown et al. 2002). Since $E \approx \epsilon$ holds for a steep electron spectrum $(\delta \approx 5$ around peak $\mathrm{p} 2$ ), the above condition can be shown to be satisfied at a scale height of $\sim 200 \mathrm{~km}$. Electrons with lower energies may be stopped higher up and produce emission in other lines (e.g., $\mathrm{H} \alpha$ ). Therefore, the similarity between the amount of energy released in the $\mathrm{D} 3$ emission and that carried by nonthermal electrons bolsters the inference that the D3 line emission during the impulsive phase is capable of revealing the electron precipitation sites. Furthermore, the D3 line has the advantage of being a density diagnostic, as compared to HXRs.

\subsection{Event Evolution in $H \alpha$}

It is also instructive to study the progression of the flare emission in $\mathrm{H} \alpha$. We measure the distance between the centroids of S1 and S2 and plot the temporal evolution of the distance in Figure 2(d). Before the HXR peak p1, S1 grows along the PIL while $\mathrm{S} 2$ shows a counterclockwise motion toward the PIL (see Figures 3(k)-(m) and the time lapse movies), which mainly 


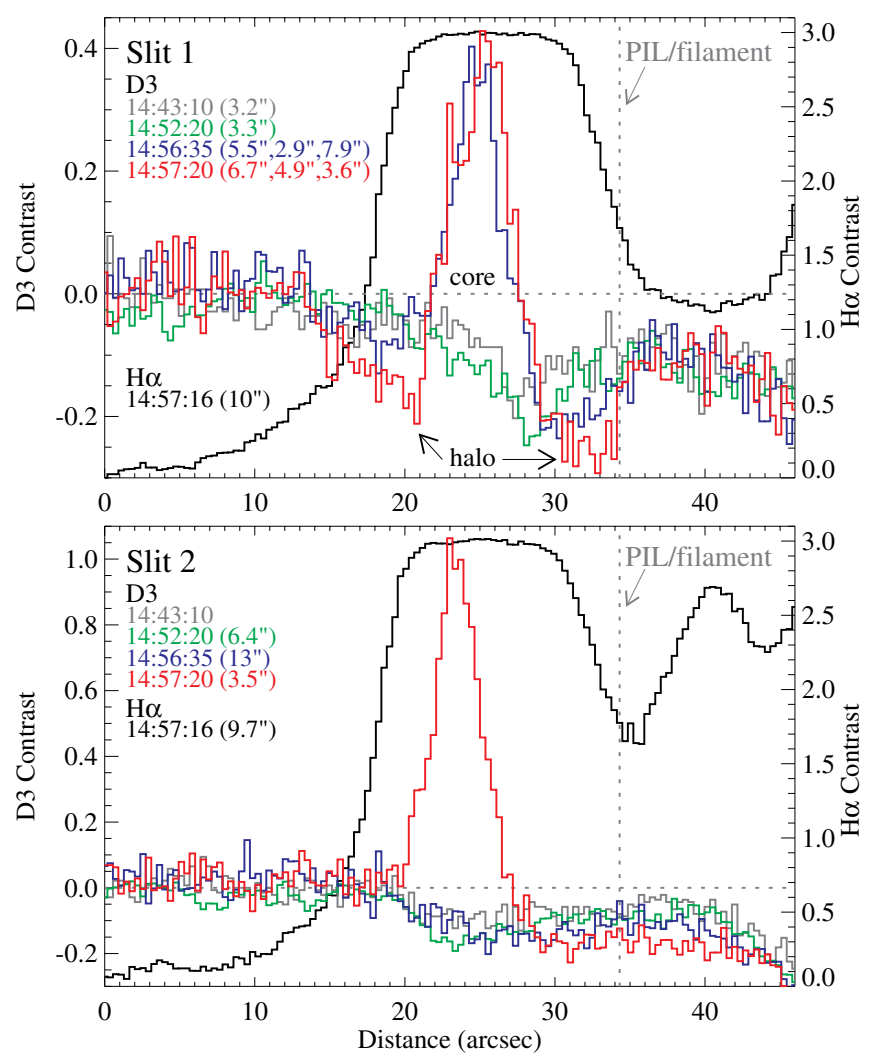

Figure 4. $\mathrm{D} 3$ and $\mathrm{H} \alpha$ intensity profiles along slits 1 and 2 (as drawn in Figure 3 ) at different times. The distance is measured from the southeastern ends of the slits. Gaussian fits are employed to derive the characteristic sizes (FWHM values are given in brackets) and peak positions of the dark halo and/or bright core emission. Note that the central $\mathrm{H} \alpha$ emission is saturated.

(A color version of this figure is available in the online journal.)

leads to a decrease of the centroid distance in the direction perpendicular to the PIL. As S1 and S2 further extend along the PIL northward and southward, respectively, the centroid distance again decreases in the direction parallel to the PIL during the HXR peaks $\mathrm{p} 2$ and $\mathrm{p} 3$. These non-standard motions of $\mathrm{H} \alpha$ ribbons distinct from the classical separation motion away from the PIL have also been observed in flares involving 3D reconnection in a fan-spine topology (Wang \& Liu 2012).

Other supporting evidence includes the following: (1) after $\sim$ 15:00 UT, the event predominantly proceeds along the PIL to form a circular outer ribbon surrounding the elongated, but more compact, inner ribbon; (2) there occurred around the HXR peak p2 a remote brightening in the northern plage region $\sim 200^{\prime \prime}$ away (see the full-disk $\mathrm{H} \alpha$ movie), which has the same positive magnetic polarity as the central spot of the flaring region; and (3) a jet-like eruption of dense material from 15:08-15:18 UT might conform to related simulations (Pariat et al. 2010). We tentatively associate the above flare ribbons/brightenings with the chromospheric mapping of the fan and spine field lines stemming from the coronal null point, as was previously done for other circular ribbon flares (Masson et al. 2009; Reid et al. 2012; Wang \& Liu 2012; Deng et al. 2013). A full investigation in this context, however, is worth pursuing in a separate study.

\subsection{Dark and Bight Circular Ribbons}

This flare is a long-duration event, since after the nonthermal impulsive phase, the GOES SXR flux decayed to its preflare level in about two hours until around 17:00 UT. The entire evolution of the event is clearly delineated by the distance-time profiles in D3 and $\mathrm{H} \alpha$ along slit 3 (Figures 5(e) and (f)), which lies perpendicular to the PIL and both the outer circular and inner compact ribbons (the white line in Figure 5). Multiwavelength profiles along the slit at a specific instance are also shown in Figure 6 . It is obvious that after expanding across the network field during the impulsive phase (cf. Figures 5(f) and 6(d)), the outer ribbon stops and remains almost fixed. The inner ribbon, however, keeps propagating northwestward during the late gradual phase at an average speed of $\sim 4 \mathrm{~km} \mathrm{~s}^{-1}$ (also see the movies). Strikingly, identical dynamics are also exhibited by the dark ribbons in D3 (Figure 5(e)). The continuous ribbon motion leads us to the conjecture that there might be persistent, moderate magnetic reconnection providing continuous injection and deposition of energy into the low atmosphere in the flare late phase. In such a stage, the energy transport mechanism is most probably thermal conduction (e.g., Czaykowska et al. 2001).

Around 15:16 UT, the NSO/KP obtained a spectroheliogram in He I $10830 \AA$. These data provide an opportunity to compare flare signatures at different heights spanning from the lower to the upper chromosphere using D3, H $\alpha$, and $10830 \AA$ images (Figures 5(a)-(c)). Several features are described as follows. (1) The circular filament, especially its southern portion, appears dark in all wavelengths. This result is evidence of heating of the dense filament materials. The jet-like disruptive activity of the southern segment around this time may be associated with the enhanced darkening in D3. (2) Postflare loops, as indicated in Figure 5 by the arrow, are dark in D3 and $10830 \AA$ while bright in $\mathrm{H} \alpha$, consistent with the extrapolation model (thick lines in Figure 5(d)). This result may indicate plasma in the postflare arcades at a temperature of $\sim 2 \times 10^{4} \mathrm{~K}$ and a density of $10^{10} \mathrm{~cm}^{-3}$ (Zirin 1980). (3) Most interestingly, the double flare ribbons are bright in $\mathrm{H} \alpha$ and $10830 \AA$ while dark in D3, which is distinctly seen as the good correspondence between the peaks in $\mathrm{H} \alpha / 10830 \AA$ and valleys in D3 of their profiles along slit 3 (Figures 6(a)-(c)). Since both the $10830 \AA$ and D3 lines are produced by ortho-helium, this result seems to imply that the $10830 \AA$ line is more easily excited than D3 and that the $10830 \AA$ line produces emission even by thermal conduction.

We explore further the dark D3 and bright $\mathrm{H} \alpha$ circular ribbons around 16:00 UT when the seeing turned excellent. It is evident that the dark ribbons in D3 and the bright ribbons in $\mathrm{H} \alpha$ demonstrate a nearly exact uniformity (cf. Figures 5(g) and (h)). Such a correlation shows up as a negative trend of ribbon pixels, as fit by the white-dashed line in the scatter plot of $\mathrm{H} \alpha$ / D3 contrast in Figure 5(i). Since the $\mathrm{H} \alpha$ emission is largely not saturated at this time, it points to a $\sim 20 \%$ D3 darkening at a $\sim 300 \% \mathrm{H} \alpha$ enhancement level, consistent with the results in Figure 4. Dark flare ribbons in D3 are rarely observed. As a comparison, the aforementioned dark D3 ring observed by Zirin (1980) has a much smaller $\left(\sim \frac{1}{7}\right)$ spatial scale and exists for only a short period of time ( $\sim 5$ minutes) in the early stage of a surge. Zirin (1980) interpreted the dark circular D3 ribbon as the projection of a shell of dense $\left(\sim 10^{10} \mathrm{~cm}^{-3}\right)$ and hot $\left(\sim 2 \times 10^{4} \mathrm{~K}\right)$ gas. Noticeably, this vision of the shell is well in line with the dome-like fan surface as we speculate for this event.

\section{SUMMARY AND DISCUSSION}

In this paper, we have presented a rare observation of a major flare in the He I D3 line, taking advantage of the recentlydigitized, high spatiotemporal resolution BBSO film data. We also compared the radiative energy in D3 at the flare peak time with the nonthermal electron energy, and discussed the event 

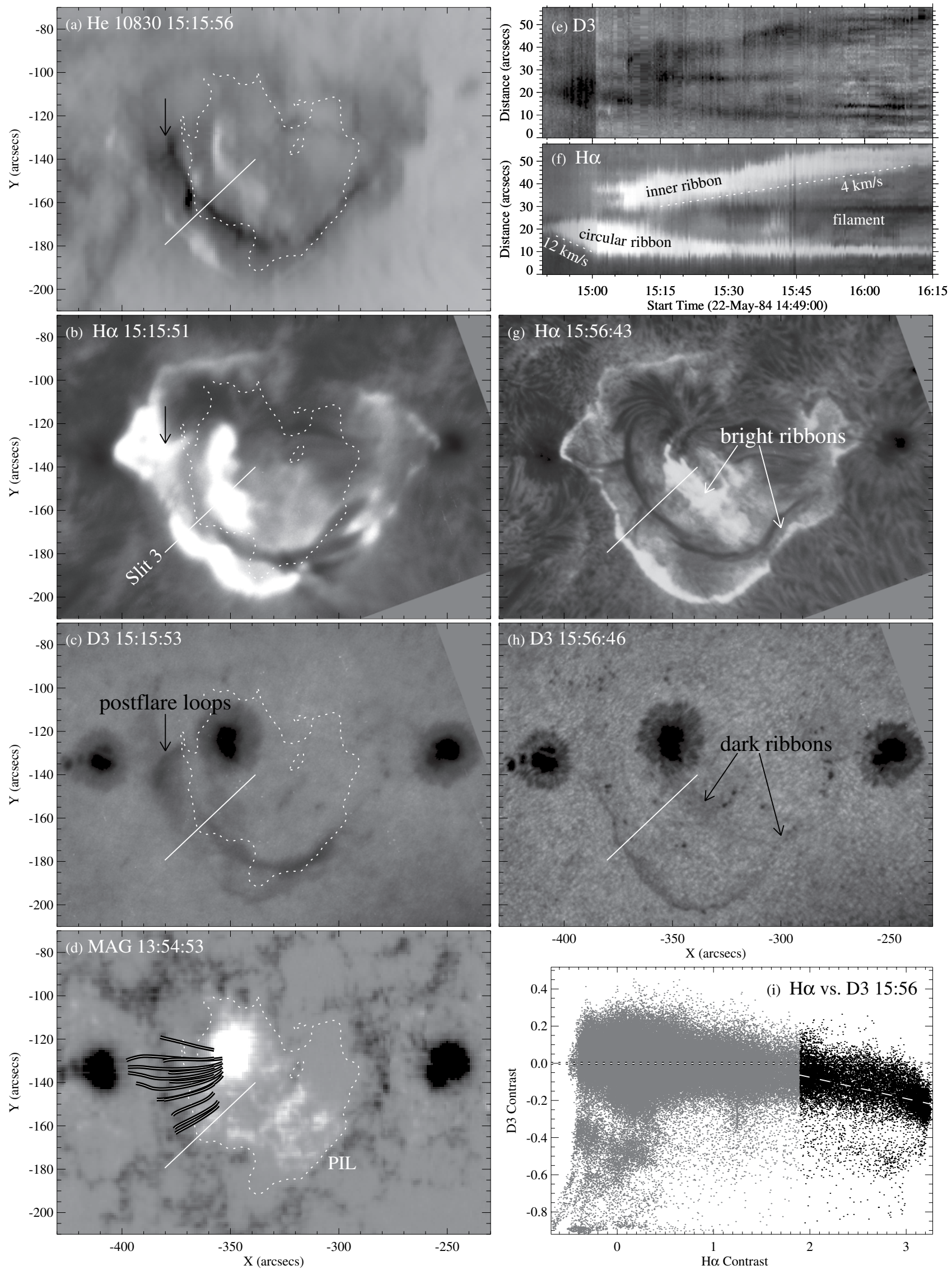

Figure 5. Dark circular ribbons in D3 ( $\mathrm{c}$ and $\mathrm{h}$ ) in comparison with their bright counterparts in $\mathrm{H} \alpha$ (b and $\mathrm{g}$ ) and He I $10830 \AA$ (a), in the late thermal phase under moderate (b and $\mathrm{c}$ ) and excellent ( $\mathrm{g}$ and $\mathrm{h}$ ) seeing conditions. The thick lines in (d) show some of the modeled potential field lines. (e) and (f) are time slices using D3 and $\mathrm{H} \alpha$ images, respectively, for slit 3 (white line). The distance is measured from the southeastern end. (i) shows the scatter plot of the H $\alpha$ contrast in (h) vs. the D3 contrast in $(\mathrm{g})$. The white dashed line is a linear fit to the black data points, which correspond to the regions of flare ribbons. 


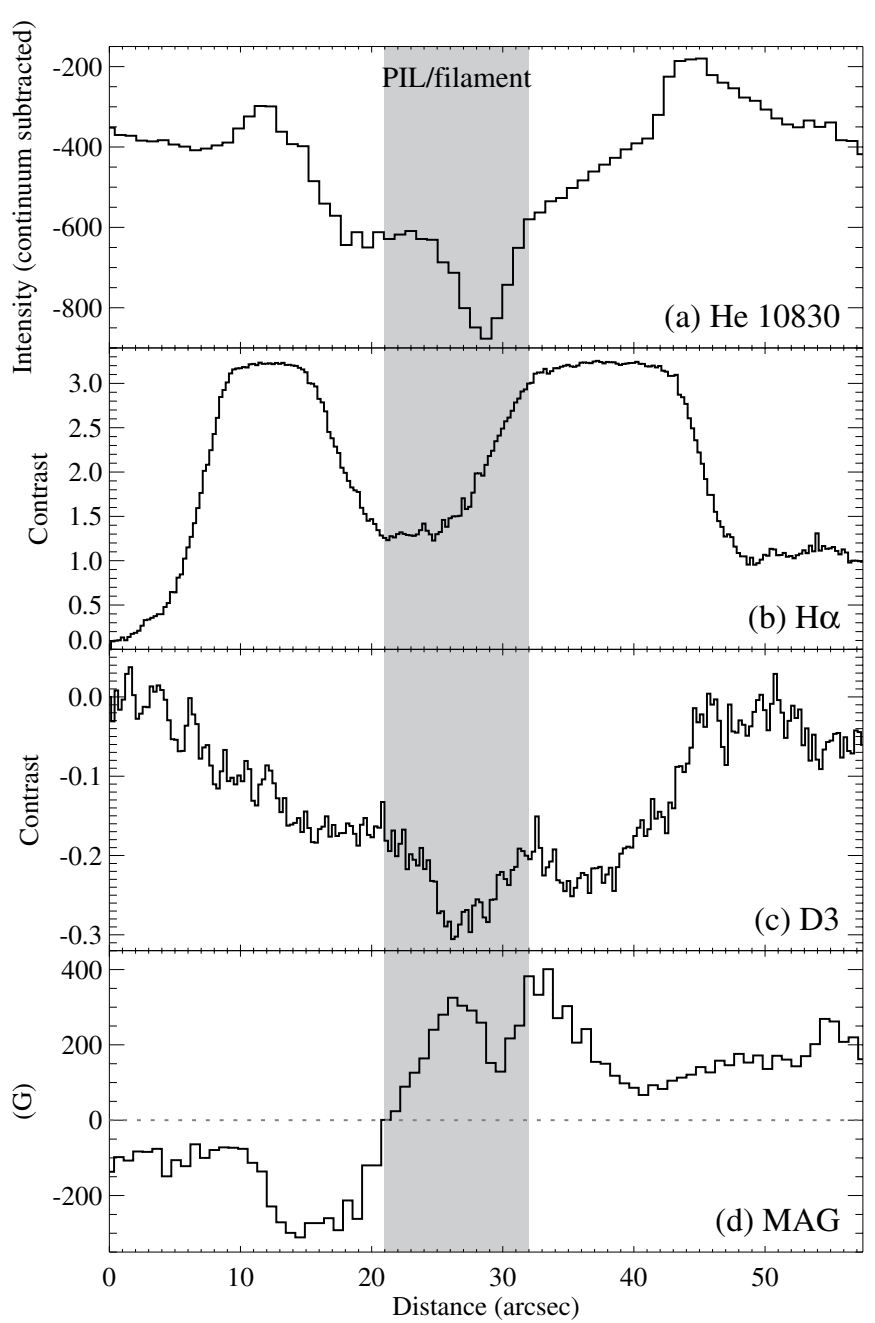

Figure 6. Spatial distribution of multiwavelength intensities and magnetic field strength at 15:15 UT along slit 3 (as drawn in Figures 5(a-d)). The distance is measured from the southeastern end. The He I $10830 \AA$ spectroheliograms of the NSO/KP were obtained by subtracting the neighboring continuum intensity from the line center intensity at each pixel. The gray region indicates the location of the PIL/filament.

evolution with the aid of $\mathrm{H} \alpha$ images and the potential field extrapolation model. Our results can be summarized as follows.

1. The flare initiation is characterized by the development of an elongated darkening source S1 in D3, which reaches a negative contrast of $\sim 5 \%$ in about four minutes (Figures 2 and 3). Within this dark source, emission kernels are seen to brighten with the first minor HXR peak. Then, simultaneous with the main HXR peak p2, they evolve into a narrow bright strand lying within the dark halo, reverting its overall contrast to positive enhancement of $\sim 5 \%$. This intensity reversal of $\mathrm{S} 1$ in $\mathrm{D} 3$ establishes this event as a rare BLF, which is highly reminiscent of those proposed in the continuum (Henoux et al. 1990).

2. The main D3 source S1 displays a bright core surrounded by dark halos (Figures 3 and 4). The bright emission core and dark absorption halos are thought to be caused by nonthermal electron heating and thermal conduction heating, respectively. This bright core-dark halo structure might be a unique characteristic of flare sources in D3 and is analogous to the bright core-halo morphology of WLFs (Xu et al. 2006).
3. The flare radiative energy loss in D3 during the $\sim 30 \mathrm{~s} \mathrm{du}-$ ration of the main HXR peak is about $10^{30} \mathrm{erg}$, which is comparable to the deposited energy of nonthermal electrons with initial energies higher than $20 \mathrm{keV}$. Together with the good correlation between the D3 and HXR time profiles (Figure 2), these results strongly suggest that D3 emission is an excellent tracer of electron precipitation in the low atmosphere during the flare impulsive phase.

4. The flare disturbance generally propagates sequentially along the PIL and ends up with a dark circular ribbon in D3 mirroring the bright ribbons in $\mathrm{H} \alpha$ and $\mathrm{He} 10830 \AA$ (Figures 3 and 5). The dark D3 ribbons in the late gradual phase indicates well the thermal conduction heating. The non-standard motions of flare ribbons and the dome-shaped magnetic structure of the flaring region (Figure 1) strongly suggest 3D magnetic reconnection in a fan-spine topology (e.g., Wang \& Liu 2012).

The mechanism for the solar continuum BLFs is different from that for the solar BLFs observed at He I D3 and $10830 \AA$. In the former mechanism, the low atmosphere first responds to the precipitation of flare electrons with increased $\mathrm{H}^{-}$opacity for a few tens of seconds at the start of the main HXR burst (Henoux et al. 1990). In the latter mechanism, the He line turns from absorption into emission when the He triplet levels are populated due to CE (for D3; Zirin 1988) and additional CR (for $10830 \AA$; Ding et al. 2005) processes. Unlike continuum BLFs that are proposed to occur before WLFs, the present D3 BLF coincides with the first small HXR peak possibly in a precursor stage before the main HXR bursts. To our knowledge, solar BLFs in the continuum and $10830 \AA$ have not yet been unambiguously observed.

A comprehensive study of the current event is unavoidably hampered by the limited quality of the aging films. We recall that flare observations at the D3 wavelength had been recognized as an important probe of the flare heating mechanism. A full understanding of the D3 signatures may also help resolve the puzzle of WLF emission in the deep photosphere (Zirin 1988). Therefore, new D3 flare observations using modern instruments and additional modeling, especially of the nonthermal effects on the D3 line, are highly desired. These efforts will shed new light on the impacts of flares in the low atmosphere.

This study is dedicated to Professor Harold Zirin, the founder of the Big Bear Solar Observatory, who passed away on 2012 January 3. We are indebted to the BBSO staff for their tremendous efforts in obtaining the D3 and $\mathrm{H} \alpha$ observations on film. We thank the referee for helpful comments. Digitization of the BBSO as well as the NSO solar film images were carried out by the Space Weather Research Lab of the NJIT, with support from NSF grant AGS 0849453 and NASA grant NNX11AC05G. The LOS magnetogram and the He $10830 \AA$ spectroheliogram used here were produced by the NSO/Kitt Peak. X-ray data were obtained by $S M M$, a NASA mission managed by GSFC, and by GOES, a NOAA/NASA project. C.L., Y.X., N.D., J.Z., and H.W. were also supported by NASA under grants NNX13AF76G, NNX13AG13G, and NNX11AO70G, and by NSF under grants AGS 1153424 and AGS 0839216. J.L. was supported by the international scholarship of Kyung Hee University and NASA grant NNX11AB49G. D.P.C. was partially supported by NSF grant AGS 0548260. 


\section{REFERENCES}

Acton, L. W., Finch, M. L., Gilbreth, C. W., et al. 1980, SoPh, 65, 53 Brown, J. C. 1971, SoPh, 18, 489

Brown, J. C., Aschwanden, M. J., \& Kontar, E. P. 2002, SoPh, 210, 373

Centeno, R., Trujillo Bueno, J., Uitenbroek, H., \& Collados, M. 2008, ApJ, 677, 742

Czaykowska, A., Alexander, D., \& De Pontieu, B. 2001, ApJ, 552, 849

Deng, N., Tritschler, A., Jing, J., et al. 2013, ApJ, 769, 112

Ding, M. D., \& Fang, C. 2000, MNRAS, 317, 867

Ding, M. D., Li, H., \& Fang, C. 2005, A\&A, 432, 699

Ding, M. D., Liu, Y., Yeh, C.-T., \& Li, J. P. 2003, A\&A, 403, 1151

Feldman, U., Liggett, M., \& Zirin, H. 1983, ApJ, 271, 832

Grinin, V. P. 1983, in IAU Colloq. 71: Activity in Red-Dwarf Stars, ed. P. B. Byrne \& M. Rodono (Astrophysics and Space Science Library, Vol. 102; Dordrecht: Reidel), 613

Hawley, S. L., Fisher, G. H., Simon, T., et al. 1995, ApJ, 453, 464

Henoux, J.-C., Aboudarham, J., Brown, J. C., van den Oord, G. H. J., \& van Driel-Gesztelyi, L. 1990, A\&A, 233, 577

Kiplinger, A. L. 1995, ApJ, 453, 973

Kundu, M. R., Nitta, N., White, S. M., et al. 1995, ApJ, 454, 522

Labonte, B. J. 1979, PhD thesis, California Institute of Technology

Lau, Y.-T., \& Finn, J. M. 1990, ApJ, 350, 672

Leibacher, J., Sakurai, T., Schrijver, K., \& van Driel-Gesztelyi, L. 2010, SoPh, 263,1

Liu, C., Lee, J., Jing, J., et al. 2010, ApJL, 721, L193
Liu, C., Lee, J., Yurchyshyn, V., et al. 2007, ApJ, 669, 1372

Livingston, W. C., Harvey, J., Slaughter, C., \& Trumbo, D. 1976, ApOpt, 15,40

Masson, S., Pariat, E., Aulanier, G., \& Schrijver, C. J. 2009, ApJ, 700, 559

Mauas, P. J. D., Andretta, V., Falchi, A., et al. 2005, ApJ, 619, 604

Najita, K., \& Orrall, F. Q. 1970, SoPh, 15, 176

Orwig, L. E., Frost, K. J., \& Dennis, B. R. 1980, SoPh, 65, 25

Pariat, E., Antiochos, S. K., \& DeVore, C. R. 2010, ApJ, 714, 1762

Reid, H. A. S., Vilmer, N., Aulanier, G., \& Pariat, E. 2012, A\&A, 547, A52

Shine, R. A., Title, A. M., Tarbell, T. D., et al. 1994, ApJ, 430, 413

Svestka, Z. 1976, in Solar Flares, (Berlin: Springer),

Tanaka, K., \& Zirin, H. 1985, ApJ, 299, 1036

Török, T., Aulanier, G., Schmieder, B., Reeves, K. K., \& Golub, L. 2009, ApJ, 704,485

van Driel-Gesztelyi, L., Hudson, H. S., Anwar, B., \& Hiei, E. 1994, SoPh, 152, 145

Vernazza, J. E., Avrett, E. H., \& Loeser, R. 1981, ApJS, 45, 635

Wang, H., Gary, D. E., Zirin, H., et al. 1996, ApJ, 456, 403

Wang, H., \& Liu, C. 2012, ApJ, 760, 101

Wang, H., Spirock, T., Goode, P. R., et al. 1998, ApJ, 495, 957

Xu, Y., Cao, W., Liu, C., et al. 2006, ApJ, 641, 1210

Zirin, H. 1975, ApJL, 199, L63

Zirin, H. 1980, ApJ, 235, 618

Zirin, H. 1988, Astrophysics of the Sun (Cambridge: Cambridge Univ. Press)

Zirin, H., \& Neidig, D. F. 1981, ApJL, 248, L45

Zirin, H., \& Tang, F. 1990, ApJS, 73, 111 